\title{
Knockdown of EPCR inhibits the proliferation and migration of human gastric cancer cells via the ERK1/2 pathway in a PAR-1-dependent manner
}

\author{
QINGLING WANG $^{1 *}$, HONGLI YANG ${ }^{1 *}$, QIAN ZHUO $^{1}$, YANYAN XU $^{1}$ and PENG ZHANG ${ }^{1,2}$ \\ ${ }^{1}$ Department of Pathology, Xuzhou Medical University, and Jiangsu Key Laboratory of Immunity and Metabolism; \\ ${ }^{2}$ Department of Pathogenic Biology and Immunology, Xuzhou Medical University, Xuzhou, Jiangsu 221004, P.R. China
}

Received August 3, 2017; Accepted February 15, 2018

DOI: $10.3892 /$ or.2018.6276

\begin{abstract}
Endothelial protein C receptor (EPCR) has been implicated in the carcinogenesis of diverse tumor types. This tumor-promoting effect of EPCR is associated with the upregulation of activated protein $\mathrm{C}$ and the activation of protease-activated receptor 1 (PAR-1). However, the exact role of EPCR in gastric cancer (GC) and the mechanisms underlying the regulation of EPCR remain elusive. In the present study, we investigated the effects of EPCR on human GC cells, as well as the underlying mechanisms. An siRNA inference system was used to knock down the expression of EPCR in GC cells, and CCK-8, colony formation and Transwell assays were performed to determine the effects of EPCR knockdown on the proliferation and migration of the tumor cells. Additionally, cell cycle distribution and apoptosis were assessed by flow cytometry, and activated PAR-1 levels were determined by cell ELISA. The results indicated that the proliferation, clonogenicity and migration were significantly reduced and that the cell cycle was arrested in the Gap 1 phase by EPCR knockdown in SGC7901 and AGS cells. Meanwhile, apoptosis was promoted by EPCR knockdown in the two cell lines. The activation of PAR-1 on the cell surface of SGC7901 and AGS cells was significantly reduced after the knockdown of EPCR. By contrast, blockade of PAR-1 reduced the proliferation and migration of gastric cells in vitro. Additionally, after the knockdown of EPCR or treatment with PAR-1 antibody,
\end{abstract}

Correspondence to: Dr Peng Zhang, Department of Pathology, Xuzhou Medical University, and Jiangsu Key Laboratory of Immunity and Metabolism, 209 Tongshan Road, Xuzhou, Jiangsu 221004, P.R. China

E-mail: pengzh@xzhmu.edu.cn

*Contributed equally

Abbreviations: EPCR, endothelial protein C receptor; PAR-1, protease-activated receptor 1

Key words: EPCR, PAR-1, proliferation, migration, ERK1/2 the expression of pERK1/2 was significantly downregulated in the SGC7901 and AGS cells, while the expression levels of p-AKT (S473) and p-AKT (T308) were unchanged. The findings of the present study demonstrated that EPCR exerts pro-carcinogenic effects in GC cells in a PAR-1-dependent manner via the ERK1/2-MAPK pathway. Thus, EPCR may be a potential molecular diagnostic or therapeutic target for GC.

\section{Introduction}

Gastric cancer (GC) is one of the main causes of cancer-related death and one of the most common epithelial malignancies worldwide, particularly in China and Japan (1). Despite decreases in the morbidity and mortality rates of GC, the overall outcomes for patients with GC have not substantially improved in recent decades (2). Thus, exploring the molecular mechanism of GC is valuable for improving the diagnosis and therapy of this disease.

Endothelial protein C receptor (EPCR) is a 46-kDa type 1 transmembrane glycoprotein. The EPCR gene consists of 4 exons and is located on chromosome 20q-11.2 (3). Evidence suggests it to be a cellular receptor for protein $\mathrm{C}$ and activated protein C (APC) (4). APC, together with its cofactor protein S, degrades factors Va and VIIIa and thereby interferes with thrombin generation and inhibits the coagulation cascade $(5,6)$. The interaction of thrombomodulin (TM) with thrombin not only changes the procoagulant substrate specificity of the protease, but also alters its proinflammatory substrate specificity by occupying exosite- 1 of thrombin, which is required for thrombin interaction and activation of protease-activated receptor 1 (PAR-1) (7,8). It has been reported that protein C activation and APC signaling pathways are localized in lipid rafts on endothelial cells and that activation of protein C-EPCR complexes by thrombin-TM complexes mechanistically links activation of the anticoagulant protein to its anti-inflammatory signaling (9). APC/EPCR interaction can directly modulate cell signaling and alter gene expression in inflammation and apoptosis (10), and can also exhibit a cytoprotective effect via PAR-1 activation (11).

EPCR primarily localizes to the endothelial cells of large blood vessels and is sporadic or absent in the microvascular endothelium of most tissues. It exists in a membrane-bound 
form or a free soluble form (sEPCR), the latter of which can regulate the quantity of circulating APC $(12,13)$. EPCR is also detected in various inflammatory cells, such as monocytes and neutrophils (10). EPCR aberrations are involved in diverse tumor types including breast cancer (14), lung cancer (15), colorectal cancer (16), ovarian cancer (17), leukemia, glioblastoma (18) and mesothelioma (19). In our previous study, EPCR was significantly upregulated in both GC tissue specimens and GC cell lines, which indicates the potential effect of EPCR in gastric tumorigenesis (20).

In the present study, knockdown of EPCR by siRNA inhibited the proliferation, clone formation and migration of SGC7901 and AGS GC cells, and arrested the cell cycle in the Gap 1 (G1) phase. In addition, knockdown of EPCR inhibited the activation of PAR-1. Treatment with the PAR-1 antibody resulted in decreased proliferation, clone formation and migration rates in SGC7901 and AGS cells. After the knockdown of EPCR or treatment with the PAR-1 antibody, the expression of pERK1/2 was significantly decreased in SGC7901 and AGS cells. These findings indicate that knockdown of EPCR inhibits the proliferation and migration of human GC cells by inhibiting the activation of PAR-1 and ERK1/2 in vitro.

\section{Materials and methods}

Cell culture. Human GC cell lines, SGC7901 and AGS cells were obtained from the Shanghai Institute of Biochemistry and Cell Biology (Shanghai, China). Cells were cultured in high glucose Dulbecco's modified Eagle's medium (DMEM) or F12 medium (both from Gibco; Thermo Fisher Scientific, Inc., Waltham, MA, USA) containing $10 \%$ fetal bovine serum $(\mathrm{FBS})$ in an incubator at $95 \%$ humidity $\left(5 \% \mathrm{CO}_{2}, 37^{\circ} \mathrm{C}\right)$.

Cell transfection. When cells reached 30-50\% confluency, they were transfected with 100 pmol anti-EPCR siRNA (sense, 5'-GCACUCGGUAUGAACUGCGGGAAUU-3' and antisense, 5'-AAUUCCCGCAGUUCAUACCGAGUGC-3') and negative control siRNA (scramble siRNA) in serum-free medium using Lipofectamine 2000 (Invitrogen; Thermo Fisher Scientific, Inc.) according to the manufacturer's instructions.

Reverse transcription-quantitative real-time polymerase chain reaction ( $R T-q P C R)$. For the RT-qPCR assay, SGC7901 and AGS cells were collected at $48 \mathrm{~h}$ after transfection and total RNA was extracted with a TRIzol reagent kit (Tiangen Biotech Co., Ltd., Beijing, China). cDNA was synthesized using a reverse transcription system kit (Vazyme Biotech Co., Ltd., Nanjing, China).

Subsequently, specific primers were used to amplify the specific gene and the qRT-PCR reaction was performed on an Applied Biosystems detection system (StepOnePlus; Applied Biosystems; Thermo Fisher Scientific, Inc.) as follows. Degeneration at $95^{\circ} \mathrm{C}$ for $30 \mathrm{sec}$, degeneration at $95^{\circ} \mathrm{C}$ for $10 \mathrm{sec}$, annealing and extension at $60^{\circ} \mathrm{C}$ for $30 \mathrm{sec}$ for a total of 40 cycles. Primers for EPCR (upstream, 5'-ACCCTGCAGC AGCTCAATG-3' and downstream, 5'-CAGGAATTCCCG CAGTTCAT-3') and GAPDH used as the internal control (upstream, 5'-AACGACCCCTTCATTGAC-3' and downstream, 5'-TCCACGACATACTCAGCAC-3') were synthesized by Shanghai Sangon Biotech Co., Ltd. (Shanghai, China). The $2^{-\Delta \Delta \mathrm{Ct}}$ method was used for analysis.

The formula was: $\Delta \Delta \mathrm{CT}=\Delta \mathrm{Ct}_{\text {experimental group }}-\Delta \mathrm{Ct}_{\text {control group }}$ where $\Delta \mathrm{Ct}=\mathrm{Ct}_{\text {target gene }}-\mathrm{Ct}_{\text {internal control }}$. $\mathrm{Ct}$ was defined as the number of amplification cycles when the optical density (OD) reached the set threshold value. All experiments were conducted in triplicate.

Western blotting. At $48 \mathrm{~h}$ after transfection, SGC7901 and AGS cells were lysed using lysis buffer (Beyotime Institute of Biotechnology, Shanghai, China). Subsequently, $30 \mu \mathrm{g}$ of total protein was subjected to sodium dodecyl sulfate polyacrylamide gel electrophoresis (SDS-PAGE) using a $10 \%$ gel, and transferred to PVDF membranes (EMD Millipore, Billerica, MA, USA). The PVDF membranes were blocked for $2 \mathrm{~h}$ at room temperature (RT) with $5 \%$ bovine serum albumin (BSA). The PVDF membranes were then incubated with mouse anti-human EPCR (cat. no. ab151403) and GAPDH (cat. no. ab9484) monoclonal antibodies (both from Abcam, Cambridge, MA, USA) at $4^{\circ} \mathrm{C}$ overnight. After rinsing 3 times with TBST ( $5 \mathrm{~min} /$ rinse), the membranes were incubated with horseradish peroxidase-labeled rabbit anti-mouse (cat. no. ab6728; Abcam) at RT for $1 \mathrm{~h}$. After rinsing 3 times with TBST, visualization was performed with an enhanced chemiluminescence method followed by exposure to X-ray film (Kodak, Rochester, NY, USA). The relative expression of protein was expressed as the gray value ratio of the target band to the internal control. The gray value of protein bands was analyzed using the ImageJ software (National Institutes of Health, Bethesda, MD, USA).

Immunofluorescence microscopy. At $24 \mathrm{~h}$ after transfection, SGC7901 and AGS cells were rinsed 3 times with ice-cold PBS and fixed in $4 \%$ paraformaldehyde, and then blocked in PBS containing 3\% bovine serum albumin (BSA; Sigma-Aldrich; Merck KGaA, Darmstadt, Germany). Cells were sequentially incubated with EPCR primary antibodies (Abcam, Cambridge, UK) and fluorescein-conjugated secondary antibodies (anti-mouse Dylight 488 conjugate; cat. no. BA1126; Wuhan Boster Biotechnology Co., Ltd., Wuhan, China). After washing with PBS, nuclei were stained with DAPI (Sigma-Aldrich; Merck KGaA). Coverslips were mounted with $90 \%$ glycerol in PBS and imaged with a fluorescence microscope (Olympus IX71; Olympus Corp., Tokyo, Japan).

Cell Counting Kit-8 (CCK-8). Transfected SGC7901 and AGS cells were reseeded in a 96-well plate at a density of 2,000 cells/well, with the viability of cells recorded at 2, 24, 48 and $72 \mathrm{~h}$. At the indicated time-points, $100 \mu \mathrm{l}$ of fresh culture solution containing $10 \mathrm{ml}$ of CCK-8 solution (Dojindo Molecular Technologies, Inc., Kumamoto, Japan) was added to each well and incubated for $2 \mathrm{~h}$ at $37^{\circ} \mathrm{C}$. The OD was detected at $450 \mathrm{~nm}$ using a microplate reader (Thermo 1500; Thermo Fisher Scientific, Inc.). All experiments were performed in 6 duplicated wells.

Colony formation assay. Transfected GC cells were reseeded in 6-well plates with DMEM or F12 at a density of 300 cells/well. After incubation for 10 days at $37^{\circ} \mathrm{C}$, cells were fixed with methanol for $2 \mathrm{~min}$ and stained with $1 \%$ crystal 
violet for $20 \mathrm{~min}$. The number of colonies consisting of more than 50 cells were counted.

Flow cytometry (FCM). After transfection for 48 h, SGC7901 and AGS cells were collected and fixed with $75 \%$ pre-cooled alcohol and stored at $4{ }^{\circ} \mathrm{C}$ overnight. After centrifugation, cells were washed with PBS twice followed by incubation with $\mathrm{RNase} \mathrm{A}$ and in a water bath for $30 \mathrm{~min}$ in the dark. Propidium iodide (PI) was used for staining, and FCM was performed to detect cell cycle distribution and to count the ratio of cells in G0/G1, S and G2/M tages by recording the red fluorescence. Experiments were repeated 3 times.

The Annexin V/7ADD double-staining method was used to detect cell apoptosis. After transfection for $48 \mathrm{~h}$, SGC7901 and AGS cells were collected at a density of $1 \times 10^{6} / \mathrm{ml}$. Then, $100 \mu \mathrm{l}$ cell suspension and $5 \mu \mathrm{l}$ Annexin V-FITC (eBioscience; Thermo Fisher Scientific, Inc.) were added into the centrifuge tube for a 15 -min reaction followed by centrifugation at $1,000 \mathrm{rpm}$ for $5 \mathrm{~min}$. Subsequently, the cells were suspended with $200 \mu \mathrm{l}$ pre-cooled binding buffer. $7 \mathrm{AAD}(5 \mu \mathrm{l})$ was added for cell staining and FCM was performed for cell cycle analysis. In the scatter diagram, healthy living cells were identified in the lower left quadrant, early apoptotic cells were in the lower right quadrant, late apoptotic cells in the upper right quadrant and necrotic cells in the upper left quadrant.

Transwell assay. After transfection for $48 \mathrm{~h}$, GC cells were transferred to an upper Transwell chamber (Corning Inc., Corning, NY, USA) with serum-free medium and 10\% FBS medium was added into the lower chamber. After incubation for $24 \mathrm{~h}$, the non-migrated GC cells were removed using a cotton swab. The cells underside of the insert were fixed in $4 \%$ paraformaldehyde for $20 \mathrm{~min}$ and stained with $1 \%$ crystal violet solution for $15 \mathrm{~min}$. Six randomized fields were examined for each insert under light microscope (Axio Scope.A1 HAL 100; Carl Zeiss, Oberkochen, Germany), with the mean value considered as an indicator for cell migration.

Cell-ELISA. After transfection for $24 \mathrm{~h}, 3 \times 10^{4}$ cells were added to each well of a flat-bottomed 96-well microtiter plate (Corning Inc.) and incubated with medium in an atmosphere of $37^{\circ} \mathrm{C}$ with $5 \% \mathrm{CO}_{2}$ overnight. The medium was then removed and the confluent cell monolayers were rinsed 3 times with washing buffer [0.01 M PBS containing $0.5 \%$ (w/v) BSA (Sigma-Aldrich; Merck KGaA)]. Non-specific binding was blocked by blocking solution containing $2 \%(\mathrm{v} / \mathrm{v})$ normal goat serum (Excell, Inc., Vincennes, IN, USA) and 1\% (w/v) BSA in $0.01 \mathrm{M}$ PBS for $1 \mathrm{~h}$ at $4^{\circ} \mathrm{C}$. The cells were subsequently incubated with the primary antibodies (anti-uncleaved PAR-1 antibody, $50 \mu \mathrm{l} /$ well) for at least $1 \mathrm{~h}$ at $4^{\circ} \mathrm{C}$ followed by the incubation of the secondary antibodies (50 $\mu \mathrm{l} /$ well) for $1 \mathrm{~h}$ at $4^{\circ} \mathrm{C}$. After washing 5 times with washing buffer, $100 \mu \mathrm{l}$ of 3,3',5,5'-tetramethylbenzidine (TMB) substrate solution (Cell Signaling Technology, Inc., Danvers, MA, USA) was added to each well and incubated for $20 \mathrm{~min}$ at RT, with the enzyme reaction stopped by the addition of $2 \mathrm{M}$ sulfuric acid. The OD of each well was measured at $450 \mathrm{~nm}$ with a multidetection microplate reader (Thermo 1500; Thermo Fisher Scientific Inc.).
The anti-uncleaved PAR-1 antibody (peptide, NH2-SFLLRNPNDKC-CONH2; peptide control, NH2-DPRSFLLRNPNDKC-CONH2) was designed and prepared by Abgent Biotechnology Co., Ltd. (Suzhou, China).

Statistical analysis. All data analysis was conducted using SPSS 16.0 (SPSS, Inc., Chicago, IL, USA). Measurement data are presented as the mean \pm standard deviation (SD). Student's paired t-test was applied to compare data between two groups. One-way ANOVA was used to compare data among three or more groups. $\mathrm{P}<0.05$ was considered to indicate a statistically significant difference.

\section{Results}

Interference efficiency of EPCR siRNA in GC cell lines $S G C 7901$ and AGS. In our previous study, we found that EPCR was significantly expressed in both GC tissues and GC cell lines (20). To determine the efficiency of inference, SGC7901 and AGS cells were transfected with the negative control siRNA and EPCR siRNA. RT-qPCR analysis revealed that the mRNA level of EPCR in the EPCR siRNA (siEPCR)transfected group was significantly decreased compared with the negative control siRNA (NC) group and the untreated control (control) group ( $\mathrm{P}<0.05$; Fig. 1A and $\mathrm{B})$. This was confirmed at the protein level by the results of western blot analysis (Fig. 1C). To ensure the location of EPCR and the inference efficiency, immunofluorescence microscopy was performed. The result indicated that EPCR was predominantly localized in the cell cytomembrane in a punctate pattern, which was downregulated after EPCR siRNA transfection (Fig. 1D).

Knockdown of EPCR inhibits the proliferation and migration of GC cells. The aberrant expression of EPCR in GC cells indicated that EPCR may be involved in the pathogenesis of GC. Hence, we investigated the effect of EPCR on the proliferation and migration of GC cells. The CCK-8 assay revealed that the proliferation of the siEPCR-transfected cells was significantly inhibited compared to that noted in the control and NC groups (Fig. 2A and B). Additionally, the colony formation assay suggested that the number of colonies in the siEPCR-transfected group was significantly decreased compared with that observed in the control and NC groups (Fig. 2C). The migration ability of the SGC7901 and AGS cells was assessed by Transwell assay after $24 \mathrm{~h}$. The number of cells that passed through the $8-\mu \mathrm{m}$ pore filter in the siEPCR-transfected group was significantly decreased compared with that noted in the control and NC groups (Fig. 2D). These results demonstrated an inhibitory effect of EPCR knockdown on the proliferation and migration of GC cells.

Knockdown of EPCR arrests the cell cycle in the G0/G1 phase and promotes apoptosis of GC cells. To investigate whether EPCR silencing could disrupt the cell cycle in SGC7901 and AGS cells, the cell cycle was analyzed by FCM using PI DNA staining. In both SCC7901 and AGS cells, the proportion of cells in the G1 phase was significantly increased in the EPCR-knockdown cells compared with the 

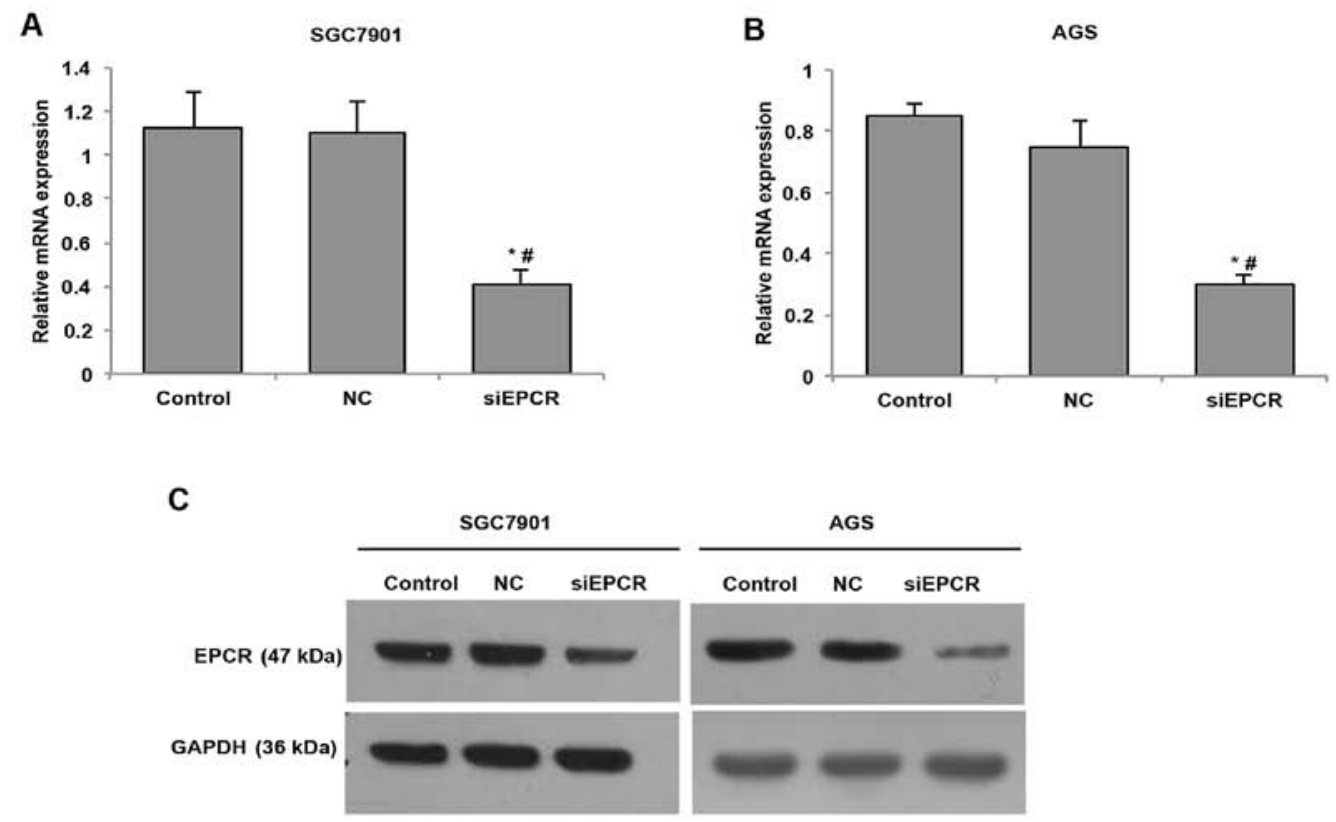

D
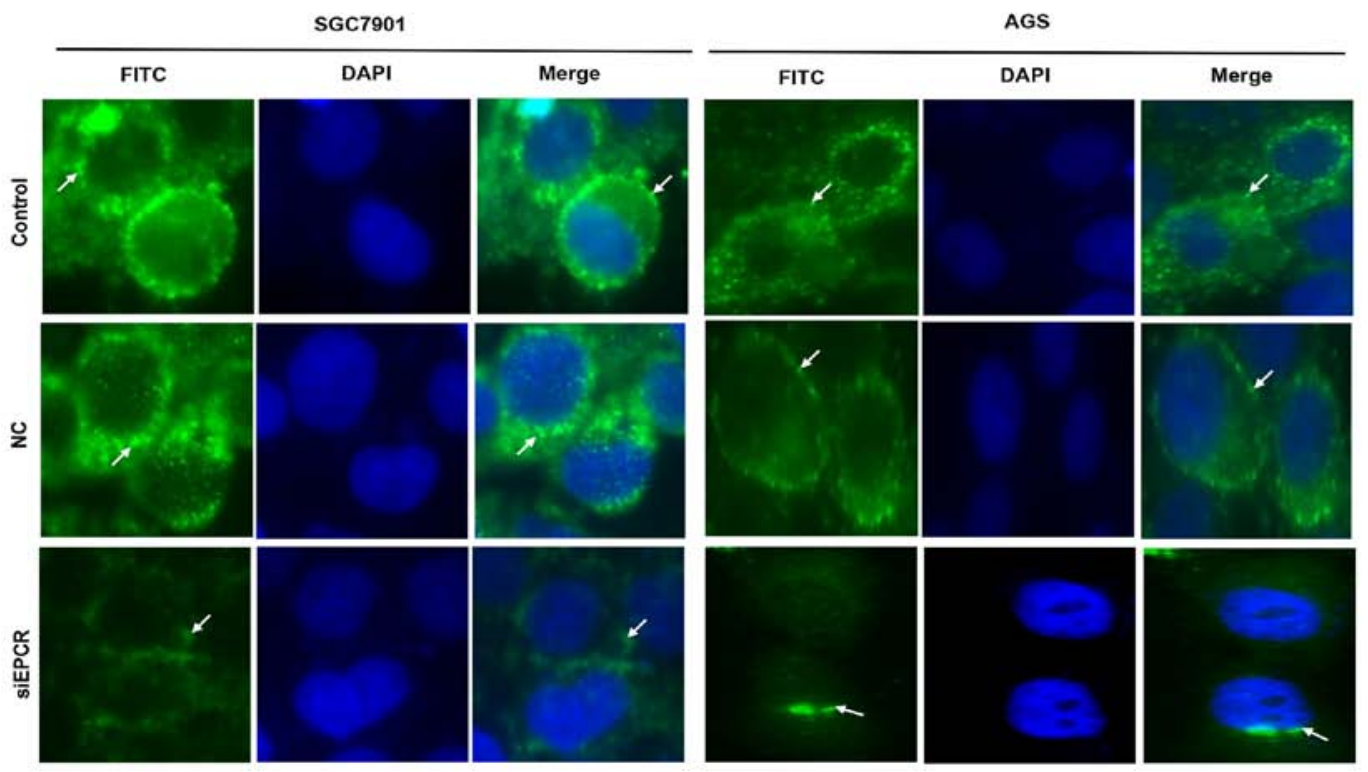

Figure 1. Interference efficiency of EPCR siRNA in SGC7901 and AGS GC cells. (A and B) The mRNA levels of EPCR in SGC7901 and AGS cells in the EPCR siRNA (siEPCR) group, negative control siRNA (NC) group and untreated control (control) group. (C) Inference efficiency of the EPCR siRNA in SGC7901 and AGS cells was determined by western blot analysis. (D) Immunofluorescence staining of EPCR (magnification, x400). The fluorescent signals of EPCR, labeled with white arrows, were detected on the plasma membrane. After EPCR siRNA transfection, the EPCR expression on the plasma membrane was downregulated compared with the NC group and control groups. Representative images are shown. All results are shown as the means \pm SD of three independent experiments. ${ }^{*} \mathrm{P}<0.05$ vs. the control group; ${ }^{*} \mathrm{P}<0.05$ vs. the $\mathrm{NC}$ group. EPCR, endothelial protein $\mathrm{C}$ receptor; GC, gastric cancer.

control and NC groups. Meanwhile, the proportions of cells in the $S$ and $G 2 / M$ phase were significantly decreased in the EPCR-knockdown cells compared with the control and NC groups (Fig. 3A-C). These findings indicated that EPCR siRNA arrested the SGC7901 and AGS cells at the G1 phase in the cell cycle.

Additionally, Annexin V/7AAD analysis showed that the rate of early apoptosis was significantly enhanced in the siEPCR-transfected group compared with that in the control and NC groups (Fig. 3D-F). This result revealed that the knockdown of EPCR expression significantly promoted apoptosis in the SGC7901 and AGS cells.
Blocking of PAR-1 inhibits the proliferation and migration of GC cells, while EPCR promotes the cleavage of PAR-1 in $G C$ cells. PARs, as transmembrane G-protein-coupled receptors (GPCRs) that are activated by a unique proteolysis mechanism, play crucial roles in vascular physiology, neural tube closure, hemostasis, and inflammation and tumorigenesis (21). EPCR and its ligand APC can promote the activation of PAR-1 and cell survival, which contributes to tumor cell endurance to stress and favors metastatic activity of lung adenocarcinoma (15). APC can aggravate the invasion and chemotaxis of tumor cells by binding to the cell surface and activating specific signaling pathways through EPCR and 

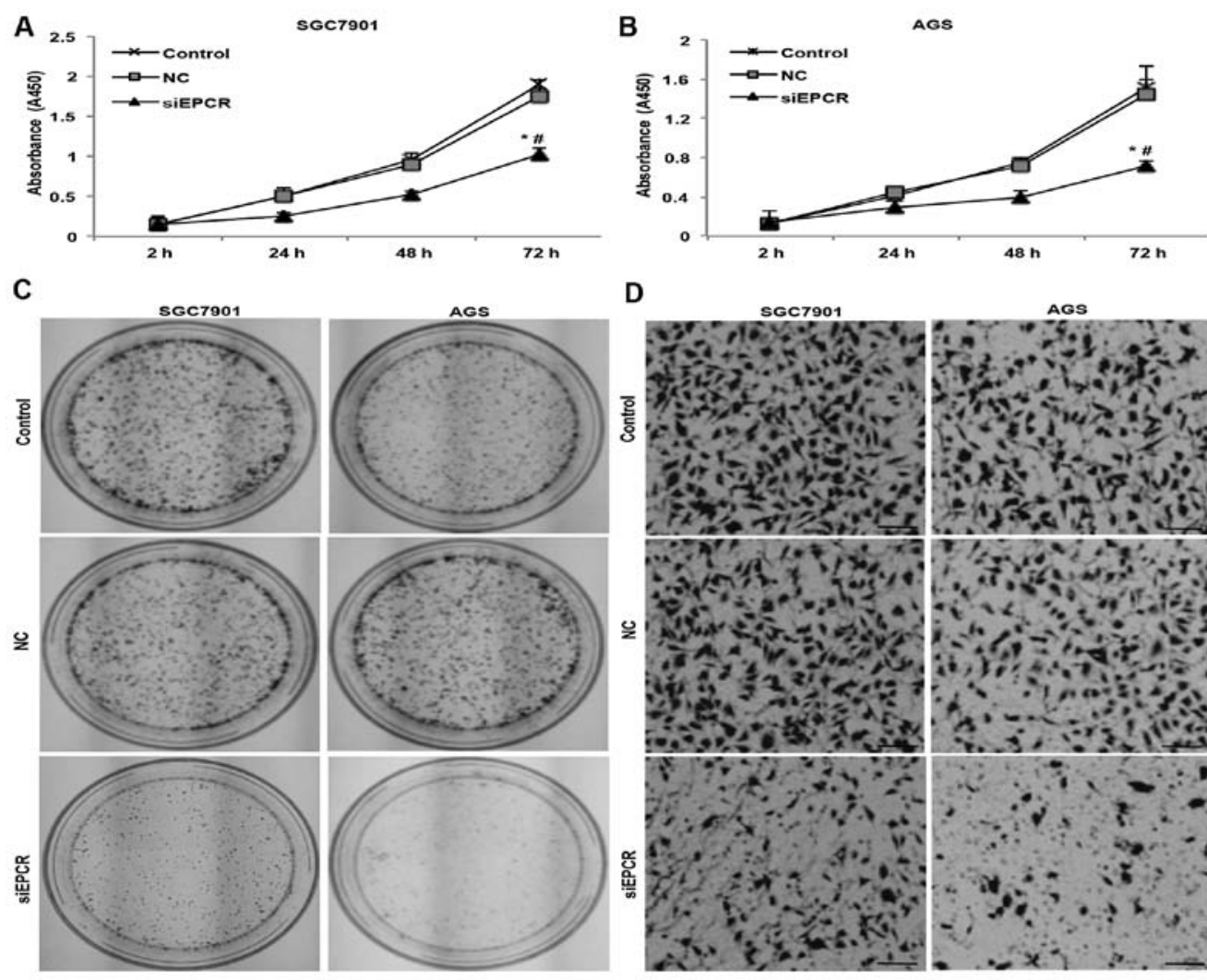

D
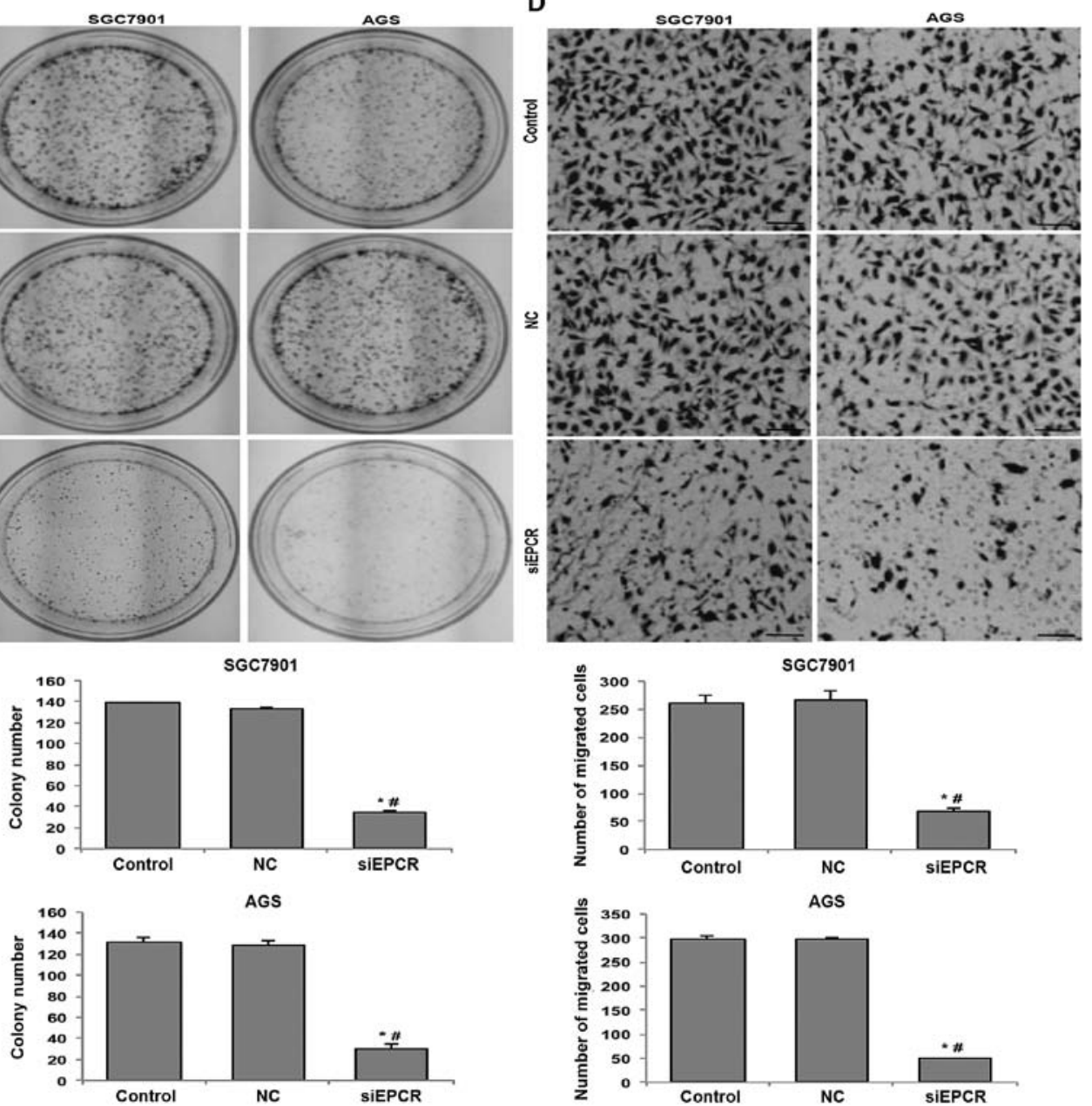

Figure 2. Knockdown of EPCR inhibits the proliferation and migration of GC cells. (A and B) Cell viability was determined by CCK-8 assay. (C) Colony formation ability was determined by colony formation assay. (D) Migration ability was determined by Transwell assay. All results are presented as the means $\pm \mathrm{SD}$ of three independent experiments. Scale bar, $100 \mu \mathrm{m}$. ${ }^{*} \mathrm{P}<0.05$ vs. the control group; ${ }^{*} \mathrm{P}<0.05$ vs. the NC group. EPCR, endothelial protein $\mathrm{C}$ receptor; GC, gastric cancer.

PAR-1 (22). Thus, we speculated that the effects of EPCR on the proliferation and migration of GC cells may be related to PAR-1. In the present study, blocking of PAR-1 with an antiPAR-1 mAb inhibited the proliferation and migration rates of the SGC7901 and AGS cells (Fig. 4A-D).

Subsequently, activation of PAR-1 following the EPCR knockdown in SGC7901 and AGS cells was investigated by a cell-based ELISA. We adopted a cell-surface ELISA using a specific PAR-1 monoclonal antibody that binds to intact but not cleaved PAR-1 in the cell-surface immunoassay. In this study, thrombin was used as a positive control, which is known to be an activator of PAR-1 (23). The results indicated that siEPCR transfection inhibited EPCR-mediated PAR-1 cleavage. The anti-uncleaved PAR-1 antibody-binding rate was increased after EPCR knockdown, compared with the control and NC groups. The anti-uncleaved PAR-1 antibody-binding rate of the thrombin-treated group was decreased compared with that noted in the control and NC groups (Fig. 5A and B). However, after EPCR knockdown, western blotting results revealed that the expression of PAR-1 was unchanged compared with the control and NC groups (Fig. 5C). These results indicate that EPCR knockdown inhibited PAR-1 activation and increased the level of uncleaved PAR-1 but did not affect the expression of PAR-1 protein in the SGC7901 and AGS cells.

Inhibition of proliferation and migration in GC cells by EPCR knockdown may be related to ERK1/2 pathways in a PAR-1-dependent manner. It was previously reported that the binding of APC with EPCR could activate PAR-1, which then phosphorylates ERK-1/2 in vascular smooth muscle cells (SMCs) (24). Additionally, APC was found to intracellularly activate ERK, AKT, and NF- $\mathrm{B}$, but not the JNK 
A
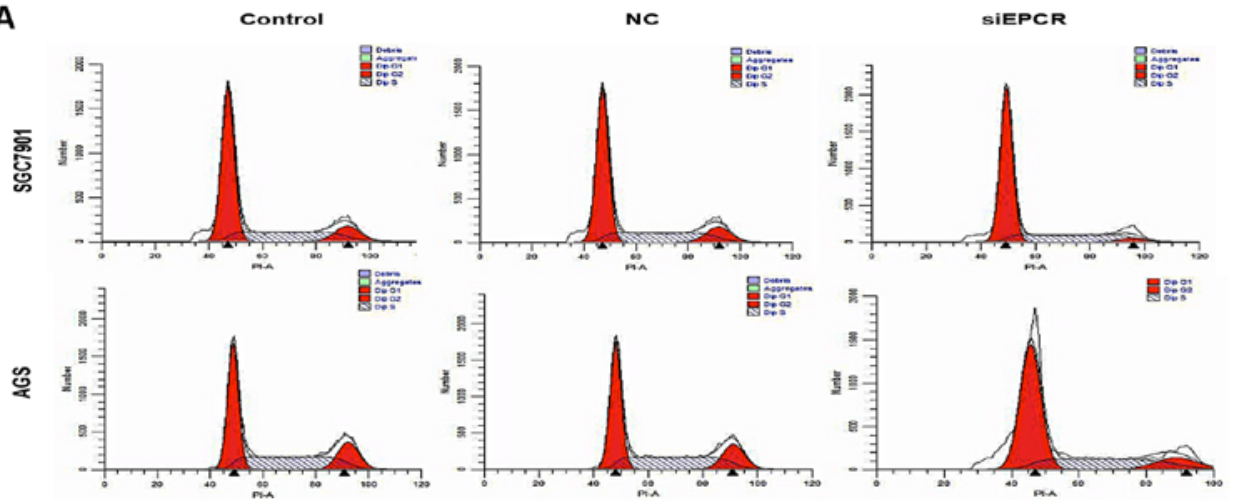

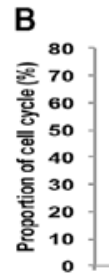
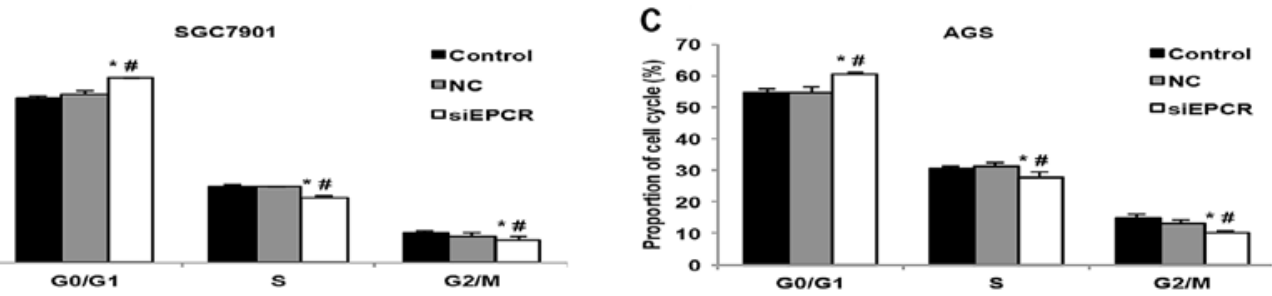

D
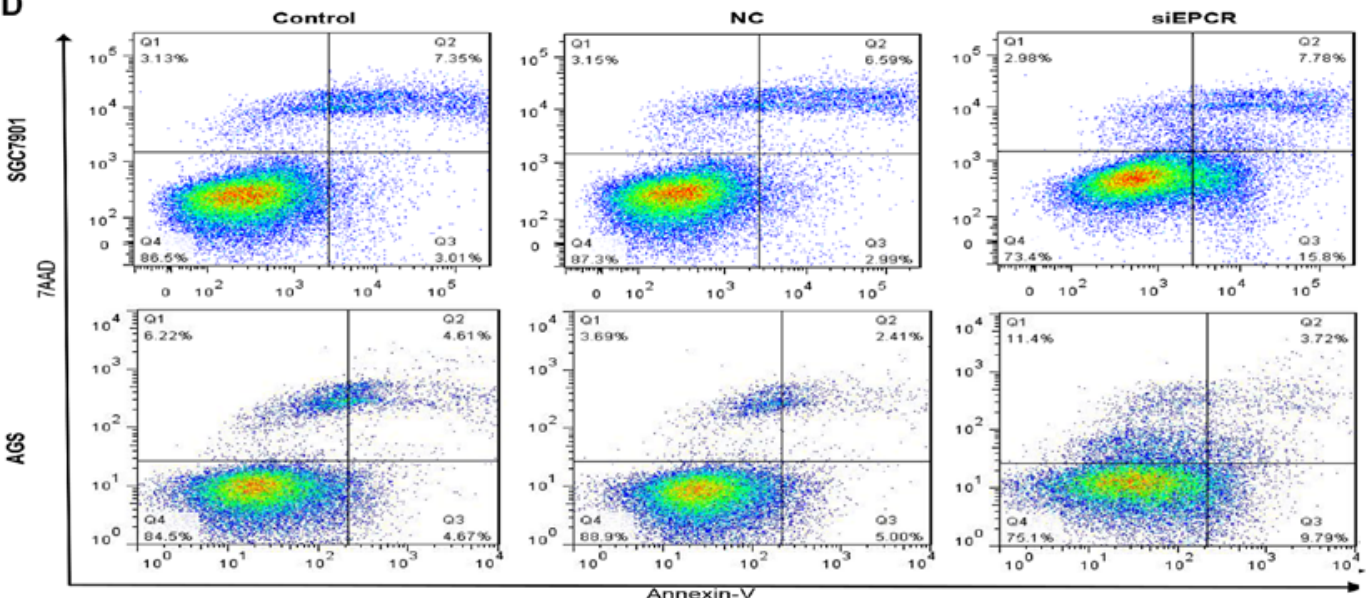

E

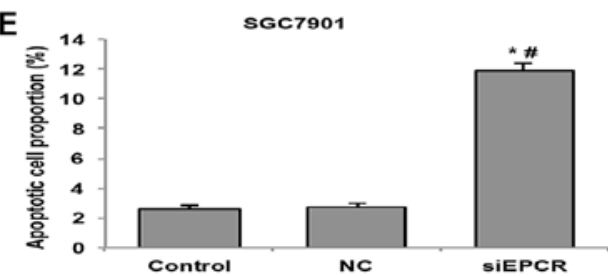

$\mathbf{F}$

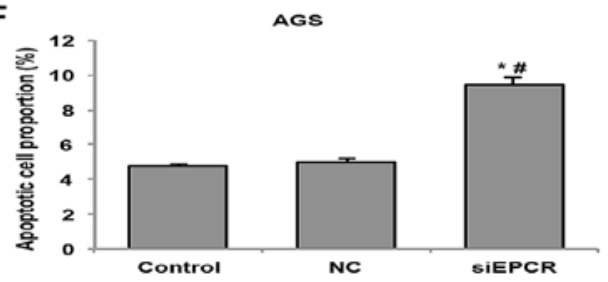

Figure 3. Knockdown of EPCR arrests the cell cycle in the G0/G1 phase and promotes apoptosis. (A-C) Cell cycle analysis of SGC7901 and AGS GC cells by FCM. (D-F) Apoptotic rates of SGC7901 and AGS GC cells detected with Annexin V/7AAD and PI staining and FCM. Experiments were performed in triplicate. All results are presented as the means $\pm \mathrm{SD}$. ${ }^{*} \mathrm{P}<0.05$ vs. the control group; ${ }^{*} \mathrm{P}<0.05$ vs. the NC. EPCR, endothelial protein $\mathrm{C}$ receptor; GC, gastric cancer; FCM, flow cytometry.

pathway, to promote MDA-MB-231 cell motility (25). To investigate whether EPCR cleavage of PAR-1 in GC cells mediates cell signaling, we evaluated the effect of EPCR on ERK1/2 phosphorylation by western blot analysis. Treatment of GC cells SGC7901 and AGS cells with EPCR siRNA decreased ERK1/2 phosphorylation, but the expression levels of p-AKT (S473), p-AKT (T308), p-JNK, and p-P38MAPK were unchanged (Fig. 6A). Furthermore, we investigated whether the activation of PAR-1 by EPCR could induce ERK1/2 phosphorylation to regulate the proliferation and migration of GC cells. Pretreatment of SGC7901 and AGS cells with monoclonal antibody against PAR-1 was observed to inhibit the phosphorylation of ERK1/2, whereas the expression levels of p-AKT (S473), p-AKT (T308), p-JNK and p-P38MAPK were unchanged (Fig. 6B). These results indicated that the inhibitory effects of EPCR knockdown on the proliferation and migration of GC cells are likely to occur via inhibition of ERK1/2 in a PAR-1-dependent manner. 

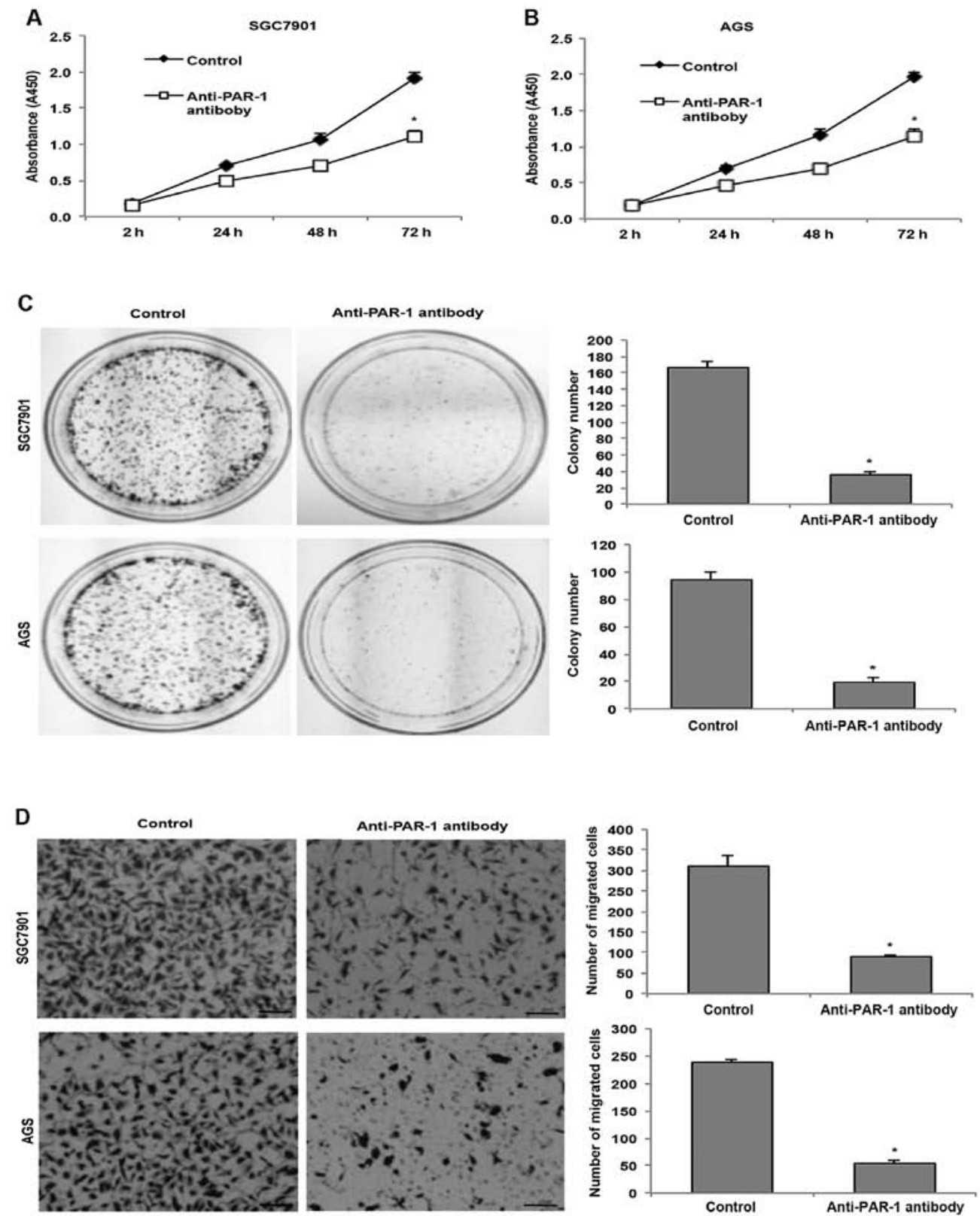

Figure 4. Blockade of PAR-1 inhibits the proliferation and migration of GC cells. (A and B) Cell viability was determined by CCK-8 assay. (C) Colony formation ability was determined by the colony formation assay. (D) Migration ability was determined by Transwell assay. SGC7901 and AGS GC cells were treated with $10 \mu \mathrm{g} / \mathrm{ml}$ anti-PAR-1 antibodies. Scale bar, $100 \mu \mathrm{m}$. ${ }^{*} \mathrm{P}<0.05$ vs. the control group. PAR-1, protease-activated receptor 1; GC, gastric cancer.

\section{Discussion}

EPCR was initially identified by Fukudome and Esmon to be localized to the endothelial cells of large blood vessels, with very low or absent expression in the microvascular endothelium of multiple tissues (3). EPCR is a key regulator of the blood coagulation system and inflammatory process $(26,27)$. Although aberrant expression of EPCR has been reported in carcinogenic processes, its exact mechanisms of action remain unclear (28). Our early data provide evidence for the presence of EPCR in GC cells and in GC tissue (20). In the present study, SGC7901 and AGS cells were used to investigate the exact role of EPCR in GC cells via knockdown of EPCR. Our data showed that EPCR knockdown significantly inhibited the proliferation and migration of the GC cells, arrested the cells in the G1 phase, and promoted apoptosis.
In addition, it was identified that the promotion of gastric tumor growth by EPCR may depend on PAR-1 and ERK1/2 activation.

EPCR is considered to exert a vascular barrier-protective effect to attenuate cancer metastasis by limiting the extravasation of tumor cells. However, overexpression of EPCR in lung adenocarcinoma cells has been reported to exacerbate metastatic activity (15). Thus, it is possible that EPCR in the endothelium of host and tumor cells may have opposing effects on cancer metastasis (29). EPCR is reportedly expressed in highly aggressive basal-like breast cancer cells and is considered to be a marker of human breast cancer stem cells (30-32). EPCR overexpression in breast cancer cells increases the growth potential of tumor cells, at least in the initial stage of tumor growth (33). EPCR also functions as a crucial negative regulator of cancer progression in 

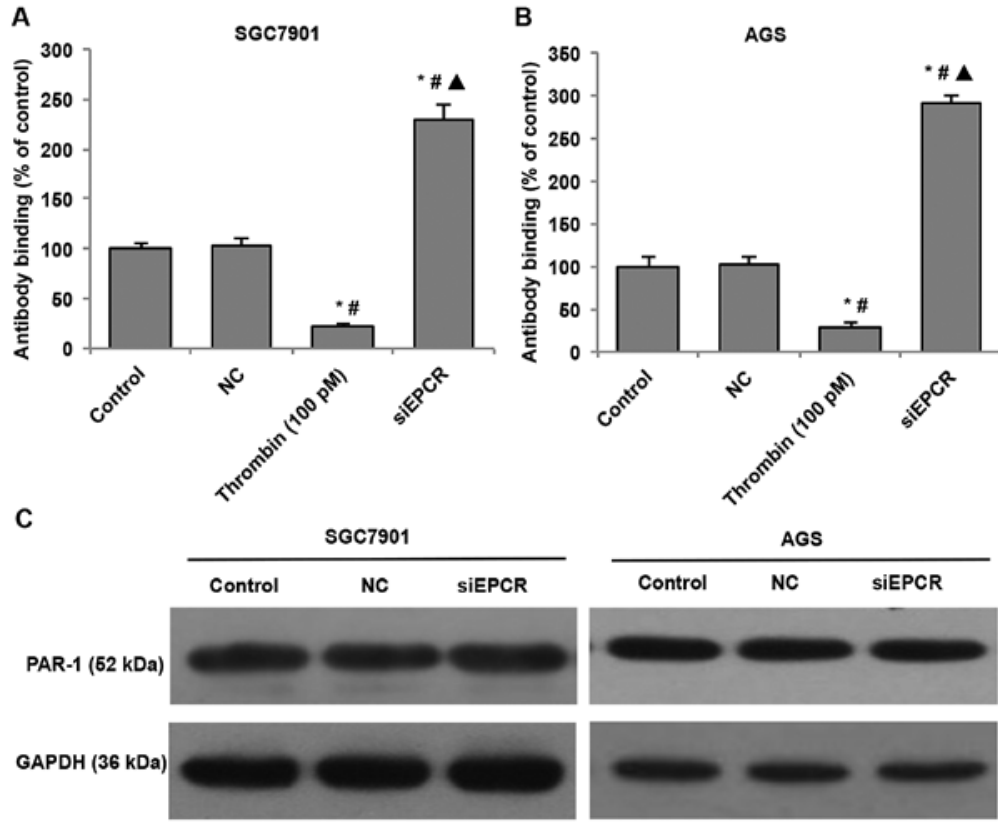

Figure 5. EPCR knockdown inhibits the cleavage of PAR-1 in GC cells. (A and B) Quantification of cell surface PAR-1 by cell ELISA after EPCR knockdown in GC SGC7901 and AGS cells (n=5). (C) PAR-1 expression was detected by western blot analysis after EPCR knockdown. All results are presented as the means \pm SD. ${ }^{*} \mathrm{P}<0.05$ vs. the control group; ${ }^{*} \mathrm{P}<0.05$ vs. the NC group; ${ }^{\wedge} \mathrm{P}<0.05$ vs. the thrombin-treated group. EPCR, endothelial protein $\mathrm{C}$ receptor; PAR-1, protease-activated receptor 1 ; GC, gastric cancer.

A

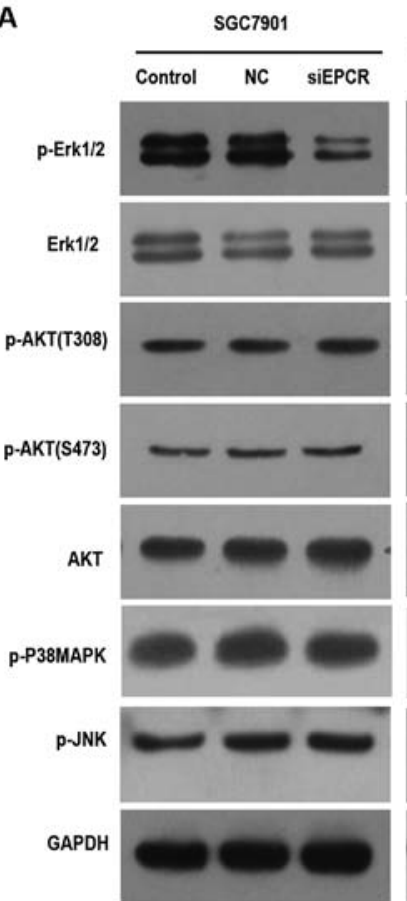

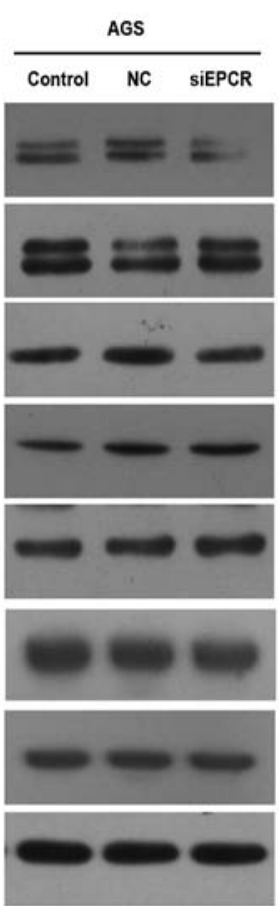

B

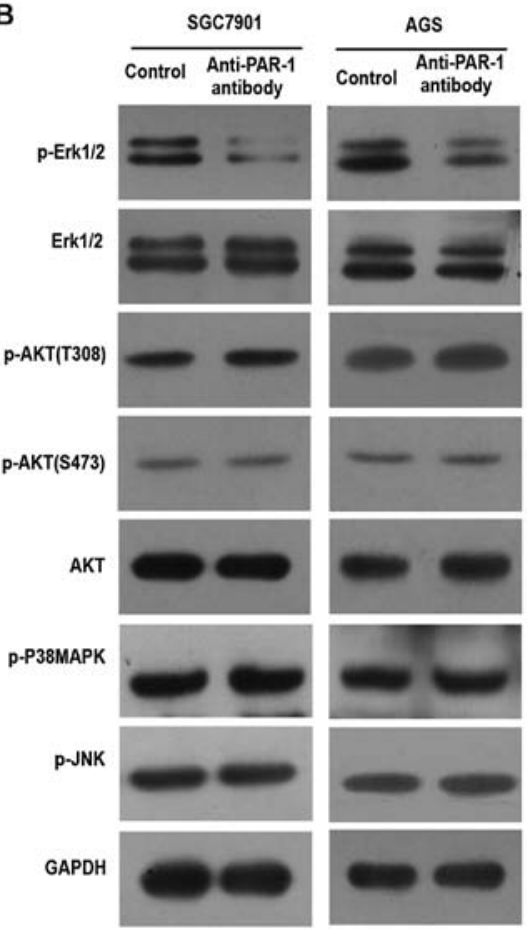

Figure 6. Knockdown of EPCR and blockade of PAR-1 inhibits the activation of ERK1/2. (A) Western blotting in SGC7901 and AGS cells showed that p-ERK1/2 were decreased in the siEPCR-transfected group, while p-AKT (S473), p-AKT (T308), p-P38MAPK and p-JNK expression levels were unchanged. (B) Western blotting in SGC7901 and AGS cells showed that p-ERK1/2 were decreased in the anti-PAR-1 antibody-treated group, while p-AKT (S473), p-AKT (T308), p-P38MAPK and p-JNK levels were unchanged. EPCR, endothelial protein C receptor; PAR-1, protease-activated receptor 1.

malignant pleural mesothelioma (MPM) and promotes tumor cell apoptosis in vivo $(19,34)$. In murine B16-F10 metastasis models, transgenic EPCR-overexpressing mice exhibited a marked reduction in liver and lung metastasis compared with wild-type animals (35). Collectively, EPCR-mediated cell signaling in a tumor cell may promote cancer cell proliferation, migration, invasion, and angiogenesis and inhibit tumor cell apoptosis $(15,25)$. Our data provide additional evidence that knockdown of EPCR may inhibit tumor progression and promote tumor apoptosis. 
Although the effect of EPCR on proliferation and migration in GC cells has been demonstrated, the mechanism underlying the effects of EPCR in GC remain unclear. EPCR was found to promote endothelial cell proliferation through the MAPK and PI3K signaling pathways $(36,37)$. In addition, APC was found to aggravate invasion and chemotaxis of breast cancer cells through EPCR and PAR-1, indicating the potential effect of EPCR on tumor cell behavior $(22,38)$. APC also intracellularly activated the ERK, AKT, and NF- $\mathrm{BB}$ pathways to promote MDA-MB-231 cell motility (25). Accordingly, we hypothesized that EPCR may activate PAR-1 and phosphorylate MAPK or AKT pathway thereafter to affect the biological behavior of GC cells. In the present study, blockade of PAR-1 predominantly inhibited the proliferation and migration of GC cells. Additionally, knockdown of EPCR significantly inhibited the activation of PAR-1, indicating that the effects of EPCR on the proliferation and migration of GC cells might be via the activation of PAR-1. Furthermore, knockdown of EPCR or blockade of PAR-1 significantly inhibited the phosphorylation of ERK1/2, but not AKT (S473), AKT (T308), JNK and P38MAPK. This indicated that the pro-carcinogenic effect of EPCR on GC cells may be attributed to the modulation of the ERK1/2 signaling pathway in a PAR-1-dependent manner in vitro.

In conclusion, EPCR exerts a pro-carcinogenic effect on GC cells in a PAR-1-dependent manner via the ERK1/2MAPK pathway, and thus may be a potent therapeutic target for GC. Notably, colocalization of EPCR and PAR-1 in lipid rafts/caveolae is reportedly required for APC to cleave PAR-1 and elicit protective cellular responses in endothelial cells (39). EPCR promotes PAR-1 and PAR-3 cleavage via APC (40). Cell membranes display a complex network of lipids and proteins designed to perform essential cell functions (41). Therefore, further studies are required to investigate whether EPCR functions through localization in the lipid rafts of gastric cells.

\section{Acknowledgements}

The authors thank ZS, HL and YZ for insightful discussion and technical assistance.

\section{Funding}

The present study was supported by the National Natural Science Foundation of China (81101493), the General Financial Grant from the China Postdoctoral Science Foundation (2014M561713) and the Dean Special Foundation of Xuzhou Medical University (2012KJZ07).

\section{Availability of data and materials}

Not applicable.

\section{Authors' contributions}

PZ participated in the design of the study. QW and HY performed most experiments and drafted the manuscript. ZQ and YX helped with cell culture and data analysis. All authors read, approved the final manuscript and agreed to be accountable for all aspects of the study in ensuring that questions related to the accuracy or integrity of any part of the work are appropriately investigated and resolved.

\section{Ethics approval and consent to participate}

The study does not require ethics approval and consent to participate.

\section{Consent for publication}

Not applicable.

\section{Competing interests}

The authors declare that they have no competing interests.

\section{References}

1. Shao Y, Sun K, Xu W, Li XL, Shen H and Sun WH: Helicobacter pylori infection, gastrin and cyclooxygenase-2 in gastric carcinogenesis. World J Gastroenterol 20: 12860-12873, 2014.

2. Jackson C, Cunningham D, Oliveira J; ESMO Guidelines Working Group: Gastric cancer: ESMO clinical recommendations for diagnosis, treatment and follow-up. Ann Oncol 20 (Suppl 4): 34-36, 2009.

3. Fukudome $\mathrm{K}$ and Esmon CT: Identification, cloning, and regulation of a novel endothelial cell protein C/activated protein $\mathrm{C}$ receptor. J Biol Chem 269: 26486-26491, 1994.

4. Ghosh S, Pendurthi UR, Steinoe A, Esmon CT and Rao LV: Endothelial cell protein $\mathrm{C}$ receptor acts as a cellular receptor for factor VIIa on endothelium. J Biol Chem 282: 11849-11857, 2007.

5. Dahlbäck B and Villoutreix BO: The anticoagulant protein C pathway. FEBS Lett 579: 3310-3316, 2005.

6. Taylor FB Jr, Peer GT, Lockhart MS, Ferrell G and Esmon CT: Endothelial cell protein $\mathrm{C}$ receptor plays an important role in protein $\mathrm{C}$ activation in vivo. Blood 97: 1685-1688, 2001.

7. Griffin JH, Zlokovic BV and Mosnier LO: Protein C anticoagulant and cytoprotective pathways. Int J Hematol 95: 333-345, 2012.

8. Rezaie AR: Protease-activated receptor signalling by coagulation proteases in endothelial cells. Thromb Haemost 112: 876-882, 2014.

9. Disse J, Petersen HH, Larsen KS, Persson E, Esmon N, Esmon CT, Teyton L, Petersen LC and Ruf W: The endothelial protein C receptor supports tissue factor ternary coagulation initiation complex signaling through protease-activated receptors. J Biol Chem 286: 5756-5767, 2011.

10. Balazs AB, Fabian AJ, Esmon CT and Mulligan RC: Endothelial protein $C$ receptor (CD201) explicitly identifies hematopoietic stem cells in murine bone marrow. Blood 107: 2317-2321, 2006.

11. Riewald M, Petrovan RJ, Donner A, Mueller BM and Ruf W: Activation of endothelial cell protease activated receptor 1 by the protein C pathway. Science 296: 1880-1882, 2002.

12. Xu J, Qu D, Esmon NL and Esmon CT: Metalloproteolytic release of endothelial cell protein $\mathrm{C}$ receptor. J Biol Chem 275: 6038-6044, 2000.

13. Shabani E, Opoka RO, Bangirana P, Park GS, Vercellotti GM, Guan W, Hodges JS, Lavstsen T and John CC: The endothelial protein $C$ receptor rs867186-GG genotype is associated with increased soluble EPCR and could mediate protection against severe malaria. Sci Rep 6: 27084, 2016.

14. Feistritzer C, Schuepbach RA, Mosnier LO, Bush LA, Di Cera E, Griffin $\mathrm{JH}$ and Riewald M: Protective signaling by activated protein $\mathrm{C}$ is mechanistically linked to protein $\mathrm{C}$ activation on endothelial cells. J Biol Chem 281: 20077-20084, 2006.

15. Antón I, Molina E, Luis-Ravelo D, Zandueta C, Valencia K, Ormazabal C, Martínez-Canarias S, Perurena N, Pajares MJ, Agorreta $\mathrm{J}$, et al: Receptor of activated protein $\mathrm{C}$ promotes metastasis and correlates with clinical outcome in lung adenocarcinoma. Am J Respir Crit Care Med 186: 96-105, 2012.

16. Scheffer GL, Flens MJ, Hageman S, Izquierdo MA, Shoemaker RH and Scheper RJ: Expression of the vascular endothelial cell protein $\mathrm{C}$ receptor in epithelial tumour cells. Eur J Cancer 38: 1535-1542, 2002. 
17. Ducros E, Mirshahi S, Azzazene D, Camilleri-Broët S, Mery E, Al Farsi H, Althawadi H, Besbess S, Chidiac J, PujadeLauraine $\mathrm{E}$, et al: Endothelial protein $\mathrm{C}$ receptor expressed by ovarian cancer cells as a possible biomarker of cancer onset. Int J Oncol 41: 433-440, 2012.

18. Tsuneyoshi N, Fukudome K, Horiguchi S, Ye X, Matsuzaki M, Toi M, Suzuki K and Kimoto M: Expression and anticoagulant function of the endothelial cell protein $\mathrm{C}$ receptor (EPCR) in cancer cell lines. Thromb Haemost 85: 356-361, 2001.

19. Keshava S, Sahoo S, Tucker TA, Idell S, Rao LV and Pendurthi UR: Endothelial cell protein $\mathrm{C}$ receptor opposes mesothelioma growth driven by tissue factor. Cancer Res 73: 3963-3973, 2013.

20. Wang Q, Liu Q, Wang T, Yang H, Han Z and Zhang P: Endothelial cell protein $\mathrm{C}$ receptor promotes MGC803 gastric cancer cells proliferation and migration by activating ERK1/2 Med Oncol 32: 162, 2015.

21. Wojtukiewicz MZ, Hempel D, Sierko E, Tucker SC and Honn KV: Protease-activated receptors (PARs) - biology and role in cancer invasion and metastasis. Cancer Metastasis Rev 34: 775-796, 2015.

22. Beaulieu LM and Church FC: Activated protein C promotes breast cancer cell migration through interactions with EPCR and PAR-1. Exp Cell Res 313: 677-687, 2007.

23. Schuepbach RA, Feistritzer C, Brass LF and Riewald M Activated protein $\mathrm{C}$-cleaved protease activated receptor- 1 is retained on the endothelial cell surface even in the presence of thrombin. Blood 111: 2667-2673, 2008.

24. Bretschneider E, Uzonyi B, Weber AA, Fischer JW, Pape R, Lötzer K and Schrör K: Human vascular smooth muscle cells express functionally active endothelial cell protein $\mathrm{C}$ receptor. Circ Res 100: 255-262, 2007.

25. Gramling MW, Beaulieu LM and Church FC: Activated protein C enhances cell motility of endothelial cells and MDA-MB-231 breast cancer cells by intracellular signal transduction. Exp Cell Res 316: 314-328, 2010

26. Scaldaferri F, Sans M, Vetrano S, Graziani C, De Cristofaro R, Gerlitz B, Repici A, Arena V, Malesci A, Panes J, et al: Crucial role of the protein $\mathrm{C}$ pathway in governing microvascular inflammation in inflammatory bowel disease. J Clin Invest 117: 1951-1960, 2007.

27. Goldenberg NM, Steinberg BE, Slutsky AS and Lee WL: Broken barriers: A new take on sepsis pathogenesis. Sci Transl Med 3: 88ps25, 2011. doi: 10.1126/scitranslmed.3002011.

28. Heng W, Mu CY, Chen C, Huang JA and Wang ZY: Endothelial cell protein $\mathrm{C}$ receptor (EPCR) is expressed by lung carcinoma and correlated with clinical parameters. Clin Lab 59: 375-380, 2013.

29. Mohan Rao LV, Esmon CT and Pendurthi UR: Endothelial cell protein $\mathrm{C}$ receptor: A multiliganded and multifunctional receptor. Blood 124: 1553-1562, 2014
30. Shipitsin M, Campbell LL, Argani P, Weremowicz S, Bloushtain-Qimron N, Yao J, Nikolskaya T, Serebryiskaya T, Beroukhim R, Hu M, et al: Molecular definition of breast tumor heterogeneity. Cancer Cell 11: 259-273, 2007.

31. Park SY, Lee HE, Li H, Shipitsin M, Gelman R and Polyak K: Heterogeneity for stem cell-related markers according to tumor subtype and histologic stage in breast cancer. Clin Cancer Res 16: 876-887, 2010.

32. Schaffner F, Yokota N, Carneiro-Lobo T, Kitano M, Schaffer M Anderson GM, Mueller BM, Esmon CT and Ruf W: Endothelial protein $\mathrm{C}$ receptor function in murine and human breast cancer development. PLoS One 8: 61071, 2013.

33. Keshava S, Kothari H, Rao LV and Pendurthi UR: Influence of endothelial cell protein $\mathrm{C}$ receptor on breast cancer development. J Thromb Haemost 11: 2062-2065, 2013.

34. Keshava S, Rao LV and Pendurthi UR: Intrapleural adenoviral-mediated endothelial cell protein $C$ receptor gene transfer suppresses the progression of malignant pleural mesothelioma in a mouse model. Sci Rep 6: 36829, 2016.

35. Van Sluis GL, Niers TM, Esmon CT, Tigchelaar W, Richel DJ, Buller HR, Van Noorden CJ and Spek CA: Endogenous activated protein $C$ limits cancer cell extravasation through sphingosine-1-phosphate receptor 1-mediated vascular endothelial barrier enhancement. Blood 114: 1968-1973, 2009.

36. Zhang Y, Hu SY, Yin T, Tian F, Wang S, Zhang Y and Chen Y: Liraglutide promotes proliferation and migration of cardiac microvascular endothelial cells through PI3K/Akt and MAPK/ERK signaling pathways. Nan Fang Yi Ke Da Xue Xue Bao 35: 1221-1226, 2015 (In Chinese).

37. Asanuma K, Yoshikawa T, Hayashi T, Akita N, Nakagawa N, Hamada Y, Nishioka J, Kamada H, Gabazza EC, Ido M, et al: Protein $\mathrm{C}$ inhibitor inhibits breast cancer cell growth, metastasis and angiogenesis independently of its protease inhibitory activity. Int J Cancer 121: 955-965, 2007.

38. Ducros E, Berthaut A, Mirshahi SS, Faussat AM, Soria J, Agarwal MK and Mirshahi M: Aldosterone modifies hemostasis via upregulation of the protein-C receptor in human vascular endothelium. Biochem Biophys Res Commun 373: 192-196, 2008.

39. Bae JS, Yang L and Rezaie AR: Receptors of the protein C activation and activated protein $\mathrm{C}$ signaling pathways are colocalized in lipid rafts of endothelial cells. Proc Natl Acad Sci USA 104: 2867-2872, 2007.

40. Bouwens EA, Stavenuiter F and Mosnier LO: Cell painting with an engineered EPCR to augment the protein $\mathrm{C}$ system. Thromb Haemost 114: 1144-1155, 2015.

41. Lingwood D and Simons K: Lipid rafts as a membrane-organizing principle. Science 327: 46-50, 2010. 